\title{
La escucha psicoanalítica y los recursos psicodramáticos en un grupo de hombres que experimentaron violencia infantil en Costa Rica
}

\author{
The Psychoanalytic Listening and the Psychodramatic Techniques in a \\ Group of Men Who Experienced Childhood Violence in Costa Rica
}

\author{
Rodrigo Zúñiga Araya ${ }^{1}$ \\ (D)https://orcid.org/0000-0003-1920-1964
}

María Laura Ortiz Arias $^{2}$

(Dhttps://orcid.org/0000-0003-1815-8001

1,2 Posgrado en Psicología, Universidad de Costa Rica, Costa Rica

\begin{abstract}
Resumen. Objetivo. Sistematizar los aportes de la escucha analítica con elementos psicodramáticos y corporales en un grupo de hombres que experimentaron violencia durante su infancia. Método. Sistematización y análisis por categorías temáticas, a partir de observación participante, registros escritos y de audio, del discurso verbal y corporal de 9 hombres en un grupo de terapia psicoanalítica con técnicas psicodramáticas. Resultados. Se concluye que la violencia promueve una relación de maltrato con el propio sujeto y la experiencia de dolor es inscrita en el cuerpo como posibilidad de expresión. El abordaje grupal facilitó la reelaboración del sufrimiento psíquico mediante el uso del cuerpo para reconocer y verbalizar emociones de la escena traumática en un encuentro de subjetividades.
\end{abstract}

Palabras clave. Violencia infantil, experiencia subjetiva, psicoterapia grupal, psicoanálisis, psicodrama, hombres.

Abstract. Objective. Systematize the contributions of the psychoanalytic clinic with psychodramatic and body techniques in a group of men who experienced violence in their childhood. Method. Systematization and analysis by theme categories, based on participant observation, written and audio recording, from the verbal and body speech of nine men in psychoanalytic group therapy by using psychodramatic techniques. Results. In conclusion, violence promotes an abusive relationship with the person himself, and the experience of pain is inscribed in the body as a possibility of expression. The group approach facilitated the reworking of psychic suffering through the body to recognize and verbalize emotions from the traumatic scene in an encounter of subjectivities.

Keywords. Childhood Violence, Subjective Experience, Group Psychotherapy, Psychoanalysis, Psychodrama, Men.

${ }^{1}$ Rodrigo Zúñiga Araya. Posgrado en Psicología, Universidad de Costa Rica. Dirección postal: 10901, San José, Costa Rica. E-mail: rodriaz29@gmail.com

${ }^{2}$ María Laura Ortiz Arias. Posgrado en Psicología, Universidad de Costa Rica. E-mail: psiconsultmarialauraoa@gmail.com

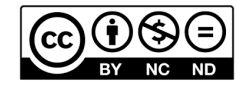

Esta obra está bajo una licencia de Creative Commons Reconocimiento-NoComercial-SinObraDerivada 4.0 Internacional. 


\section{Introducción}

La violencia infantil afecta al menos mil millones de niños y niñas alrededor del mundo, la mitad de toda la población infantil (Naciones Unidas, 2019); dichas cifras coinciden en Costa Rica donde casi la mitad de las personas menores de edad ha sido víctima de violencia de acuerdo con el Ministerio de Salud, el Instituto Nacional de Estadística y Censos (INEC) y el Fondo de las Naciones Unidas para la Infancia (UNICEF, 2018).

Los estilos de crianza y la autoridad parental justifican muchos actos de violencia en la infancia, en tanto que las figuras parentales los legitiman como formas de disciplina válidas. Miller (1998; 2002; 2009) afirma que la validez social y cultural normalizan el castigo físico que se reproduce y se transmite de una generación a otra. Se justifica y tanto la persona menor de edad como la adulta no lo considera como violencia, aunque los primeros guardan la queja.

En ese sentido, Uribe (2010) menciona que el maltrato como estrategia de crianza es inútil, ya que el sujeto prioriza la queja sobre el daño causado en su cuerpo o sentimientos y no reflexiona sobre la falta cometida. Por tanto, desde Freud se enseñaba que la violencia intrafamiliar se origina en dificultades para tramitar la agresividad propia adecuadamente. Si bien aún los psicoanalistas post freudianos no conceptualizan el maltrato como tal, sino como traumas tempranos preedípicos por fallas de la función materna, estos generan déficits en la formación del yo (Uribe, 2010); lo relacionan con frustraciones de demanda de amor del niño hacia sus figuras paternas y el deseo de ser reconocido por el Otro (el orden simbólico) (Uribe, 2010). Más aún, el maltrato infantil, físico y psicológico se transmite sobre la base del miedo y afecta el desarrollo de herramientas psicoafectivas relacionadas con la adaptación, la autonomía y el sentido de independencia necesaria para la toma de decisiones y la acción (Rosabal, 2009; Cuervo, 2010).

Las víctimas de violencia en la niñez por sus cuidadores crecen pensando que tienen alguna carencia y pueden culparse por el abuso sufrido, internalizando agresiones físicas y verbales, además de presentar baja autoestima y dificultad para relacionarse (UNICEF, s.f.; Organización Mundial de la Salud, 2020) y aumento de riesgo de comportamientos autodestructivos en la vida adulta (Hardt et al., 2008; Evren et al., 2011; Carlson et al., 2013).

Se puede distinguir entre conducta autodestructiva directa e indirecta. El primero es un comportamiento autolítico consciente y deliberado, como el suicidio; el segundo se refiere a formas sub-intencionadas que podrían precipitar el riesgo de muerte de modo inconsciente, como conductas temerarias (Clemente \& González, 1996). Tsirigotis et al. (2013) plantearon cinco tipos de estos últimos; a saber, transgresión y riesgo, mal mantenimiento de la salud, negligencia personal y social, falta de planeamiento y pasividad e impotencia. A su vez, encontraron que los hombres se caracterizan por alta "autodestructividad indirecta".

Esto puede relacionarse con los patrones socioculturales de construcción de la identidad masculina asociada con una alta predisposición a los comportamientos autodestructivos indirectos, como comportamientos de riesgo y transgresivos, así como un sentido de impotencia (Campos \& Salas, 2002; Burin \& Meler, 2004; Salas, 2005; Butler, 2007). Se trata de un modelo de masculinidad que enseña a no expresar los sentimientos de vulnerabilidad (Campos \& Salas, 2002) y a cumplir una serie de marcadores de virilidad para medir a los hombres y dar prueba de su virilidad (Gilmore, 1994). Dichas exigencias pueden provocar "angustia, dificultades afectivas, miedo al fracaso y comportamientos compensatorios potencialmente peligrosos y destructores" (Badinter, 1993, p. 174).

Actualidades en Psicología, 35(130), 2021, 75-95 
En efecto, en Costa Rica, los datos indican que por cada mujer que muere en accidentes viales 7 hombres lo hacen, mientras que más del $80 \%$ de las víctimas por homicidio son hombres (Comisión Técnica Interinstitucional sobre Estadísticas de Convivencia y Seguridad Ciudadana, 2019). De igual forma, un $80.7 \%$ de suicidios corresponde a la población masculina (Poder Judicial, 2019; Molina, 2019), cifra similar en todo el mundo.

En relación al cuerpo como destino de la autoagresión, Lema (2014) plantea que aquello que no tiene lugar en la palabra se encuentra en el cuerpo en donde se encuentran algunos posibles efectos del estrago materno, a saber: impulsividad, toxicomanías, distorsiones de la imagen corporal, entre otros. Además, siempre ha habido una inscripción simbólica y literal en el cuerpo que "producen y reproducen cuerpos haciéndolos objetos de clasificaciones, categorizaciones, modas, prohibiciones, prescripciones" (Fernández, 2001, p. 266).

Por tanto, el cuerpo del que habla el psicoanálisis resulta significativo, ya que se refiere "de un cuerpo que habla, o cuerpo hablado, al menos de un cuerpo que habrá de manifestarse deviniendo palabra" (Fernández, 2001, p. 264). Se plantea que este cuerpo del que se habla es uno que se hace presente en función de la aparición del síntoma, como forma de lenguaje.

Precisamente, Freud planteaba que "la compulsión de repetición devuelve también vivencias pasadas que no contienen posibilidad alguna de placer, que tampoco en aquel momento pudieron ser satisfacciones..." (1920/1976, p. 20). Así, el paciente repite lo que no consigue recordar, de forma que repite/actúa lo reprimido como una vivencia actual para no recordarlo (Freud, 1914/1976). La hostilidad o pulsiones destructivas hacia los objetos exteriores retornan y se dirigen contra sí mismo (Freud, 1915-1917/1976; Miller, 1998; Miller, 2009).

Para Freud, el trauma "se trata de un acontecimiento que altera una regulación y no puede explicarse" (1932, citado en García, 2005, p. 10); no lo refiere a algo extraño que aparece de la nada, sino a lo familiar que se vuelve extraño ante un evento externo, por lo cual plantea el concepto de lo ominoso, entre la familiaridad y la extrañeza. Es decir, para Freud el trauma sería el encuentro de una fantasía interna anudada con un acontecimiento del exterior (Freud, 1932 citado en García, 2005).

Por su parte, Lacan indica que en el trauma no hay motivación sino repetición y lo describe como un evento que no ha sido articulado en un mito (2003, citado en García, 2005); por cuanto, un acontecimiento actual o vivido será traumático conforme toque lo real de un acontecimiento anterior. Se refiere a algo que, antes desaparecido, irrumpe, reaparece, cambiando la organización simbólica que tenía. De esta forma, para él el trauma es "un hecho real articulado en un lenguaje" (1992, García, 2005, p. 37). Nos habla de un agujero donde no hay palabras, no obstante, "hace hablar, ordena el discurso" (García, 2005, p. 56).

Para Lacan, el trauma, como encuentro con lo real que irrumpe, solo puede cambiarse mediante un forzamiento sobre el lenguaje, lo simbólico, para cambiar el sentido imaginario (1993, citado en García, 2005). Más adelante, el autor afirma "el cuerpo, si lo tomamos en serio, es el primero que puede llevar la marca propia que lo ordena en una serie de significantes" (Lacan, 2012, p. 432.). Nos habla así de un anudamiento entre lenguaje y cuerpo en relación con las marcas que deja el trauma.

Así, Freud señalaba que las palabras pueden estar ligadas a algunos afectos, por cuanto buscaba desligar y volver a ligar cargas de afecto; desde una postura post freudiana, no 
solo surge la posibilidad de anudar lo simbólico, lo imaginario y lo real, sino la relación que existe entre el lenguaje y el cuerpo en el trabajo en experiencias traumáticas (García, 2005). En este sentido, se considera necesaria una escucha psicoanalítica que tome en cuenta el discurso del sujeto, no obstante, pueda interactuar con terapias orientadas en el cuerpo para poder trabajar en el trauma y el conflicto (Beutel et al., 2008) y advenir la subjetividad.

En términos técnicos psicoanalíticos, se parte principalmente de la asociación libre de ideas, la transferencia, la atención flotante (Freud, 1913/1976), así como la interpretación (Freud, 1914/1976) como ejes para llegar al insight. Freud resalta entonces el hacer hablar al sujeto, regla fundamental, dar importancia a la transferencia en tanto resistencia y comunicar o interpretar hasta que haya una transferencia operativa (Freud, 1913/1976).

En este sentido, la propuesta terapéutica a continuación, se basa en la psicoterapia breve de orientación psicoanalítica (Braier, 1999), la cual define algunas normas en relación con dicha técnica. La asociación libre se realiza limitándose a lo relacionado con los problemas definidos para abordar y la interpretación se restringe a resistencias transferenciales. A su vez, se proponen objetivos específicos o limitados y focaliza el abordaje a una sintomatología o problemática determinada, así como a un tiempo fijado y corto.

Asimismo, se consideran igualmente las entrevistas preliminares (Miller, 2006; Aulagnier, 2003; Braier, 1999) cuyos objetivos son la evaluación diagnóstica, la introducción del inconsciente, la localización y rectificación subjetiva en torno a la queja, así como la instauración de la transferencia y el conocimiento de la demanda. Estos corresponden al "tratamiento de ensayo" que Freud establecía como periodo de ensayo con un objetivo diagnóstico (Freud, 1913/1976).

No obstante, no se parte de "el" dispositivo analítico como si fuera único, haciendo eco de la reflexión de Rousillon sobre "una teoría general de dispositivos analíticos" (1995, citado en Rache, 2015, p. 13); se apoya del saber del psicoanálisis y busca las posibilidades de contar con nuevas clínicas que den cuenta igualmente del sujeto, por cuanto se toman elementos psicodramáticos de orientación lacaniana (Herrera, 2009).

Rousillon plantea que "si la apropiación subjetiva por la simbolización representa la 'finalidad' del trabajo psicoanalítico, entonces el dispositivo debe poder 'simbolizarlo'; debe poder simbolizarlo 'en acto', 'de hecho', en su construcción y su utilización" (1995, citado en Rache, 2015, p. 14). Por tanto, las técnicas psicodramáticas y corporales funcionan como herramientas complementarias de la escucha analítica (Reyes, 2007). Así, el registro de lo imaginario "se inscribe la práctica terapéutica del psicodrama, [mientras] lo simbólico es en psicodrama, más que un medio, un fin, un producto" (Herrera, 2009, p. 2), como en el psicoanálisis.

El psicodrama se desarrolla a través de lo corporal, vincular, escénico, metafórico y creativo para activar un cambio y una resignificación. Asimismo, hace uso de la "transferencia" que, a pesar de algunas diferencias con el concepto freudiano, se refiere a la depositación en el otro (el semejante), mediante el vínculo, de objetos primarios introyectados. Se toma el cuerpo como texto verbal con memorias reprimidas y que pueden desplegarse en lo escénico a través de la espontaneidad y de una serie de técnicas que conjugan lo verbal y lo no verbal, la palabra y el cuerpo (Reyes, 2005).

El psicodrama implica un territorio, escenarios, ritualización y encuadre del dispositivo con efectos de marca dramática: "es una terapéutica que intenta llevar al sujeto a ser artífice de su propio texto, descubridor de su propia verdad" (Pavlovsky, 1991, p. 158). Este autor trabaja la resistencia, por ejemplo, mediante concretización de imágenes 
(traer a escena miedos que obstaculizan), inversión de roles y el diálogo. En esa línea, Buchbinder y Matoso destacan la temática del cuerpo y la fantasmática corporal (1993, citados en Reyes, 2007) y desarrollan el trabajo con metáforas por medio de máscaras y del mapa fantasmático.

Para Arévalo et al. (2016), mientras el relato puede generar una reflexión desde lo cognitivo, con el cuerpo tal reflexión viene del movimiento mediante la transferencia; de este modo, surge una memoria corporal y emocional reprimida o no apalabrada (Miller, 2009), por cuanto se requiere pasar de la palabra al cuerpo y de este de nuevo a la palabra.

\section{Método}

El tipo de investigación es cualitativo y descriptivo, cuyo diseño es fenomenológicohermenéutico (Sampieri et al., 2014), que busca explorar la experiencia de maltrato vivida por hombres en su niñez a partir de una propuesta de psicoterapia grupal.

\section{Participantes}

La muestra fue no probabilística y autoseleccionada debido a los propósitos de la investigación, la cual se basa en la cantidad de 10 casos recomendados para estudios fenomenológicos, según Sampieri et al. (2014). La muestra se conformó de manera que se hizo una invitación abierta para hombres que asistían a los grupos de apoyo del Instituto WËM en Costa Rica; de este modo, se anotaron 25 hombres, de los cuales 10 no contestaron o no asistieron a la entrevista preliminar, 4 tuvieron criterios de exclusión y 2 no se presentaron a las sesiones grupales, así se conformó un grupo de 9 hombres en total.

Los criterios de inclusión fueron los siguientes:

- Ser hombre, mayor de 18 años.

- Tener una historia de violencia en su infancia por parte de sus figuras de crianza.

- Tener un mínimo de tres sesiones en el Grupo de Apoyo del Instituto WËM.

- No encontrarse en un escenario de crisis o emergencia actual.

- Estar anuente a participar de una entrevista preliminar.

- Tener disponibilidad de asistir a una sesión por semana.

Los participantes eran hombres de 25 a 58 años, de los cuales 3 se encontraban en relación de pareja, 2 solteros y 4 separados, mientras que de ellos 6 eran padres. En el ámbito laboral 2 eran administradores, 1 ingeniero, 1 informático, 1 estudiante universitario con empleo ocasional, 1 diseñador digital y 2 del sector industrial (Tabla 1).

\section{Diseño terapéutico}

Se diseñó un plan terapéutico, el cual se basó en la psicoterapia breve de orientación psicoanalítica (Braier, 1999). Se recurrió, además, del apoyo de elementos psicodramáticos de orientación lacaniana (Lemoine \& Lemoine, 1979, citados en Herrera, 2009) con el fin de realizar un diseño que diera cuenta de lo grupal, lo verbal y lo corporal, así como de una lectura psicoanalítica menos ortodoxa, al no existir un modelo breve que los integrara. En ese sentido, a parte del discurso, se realizaba 1 o 2 ejercicios psicodramáticos por sesión, los cuales surgían espontáneamente (Reyes, 2005) según la necesidad del grupo y a partir de algún significante expresado; se consideró que una planeación anterior podría limitar y sesgar el dispositivo psicoterapéutico. 
Tabla 1

Descripción de los participantes

\begin{tabular}{|c|c|c|c|}
\hline Seudónimo & Edad & Ocupación & Dificultades en la vida adulta \\
\hline "Iván" & 53 años & Comerciante & $\begin{array}{l}\text { Violencia en relaciones de pareja, alcohol, drogas, } \\
\text { ideaciones suicidas }\end{array}$ \\
\hline "Isaac" & 38 años & Administrador & $\begin{array}{l}\text { Comportamiento impulsivo hacia los demás y ha- } \\
\text { cia sí mismo, drogas, ansiedad, ideaciones suicidas }\end{array}$ \\
\hline "Camilo" & 29 años & Ingeniero & $\begin{array}{l}\text { Violencia por parte de su pareja, incapacidad de } \\
\text { tomar decisiones }\end{array}$ \\
\hline "Antonio" & 52 años & Administrativo & $\begin{array}{l}\text { Arrebatos de enojo y violencia psicológica en } \\
\text { contra de su pareja }\end{array}$ \\
\hline "Francisco" & 33 años & Informático & $\begin{array}{l}\text { Violencia por parte de su pareja y dificultad en la } \\
\text { toma de decisiones }\end{array}$ \\
\hline "Rafael" & 45 años & Área industrial & Intento de suicidio, alcohol, drogas y delincuencia \\
\hline "Mateo" & 37 años & Estudiante & Depresión, ideación suicida, alcohol, drogas \\
\hline "Leonardo" & 58 años & Sector industrial & $\begin{array}{l}\text { Violencia a familia, intento de suicidio, problemas } \\
\text { con la ley, alcohol, drogas }\end{array}$ \\
\hline "Javier" & 25 años & Diseñador & Ansiedad, ira, ideación suicida \\
\hline
\end{tabular}

Los ejercicios psicodramáticos, los cuales son descritos brevemente en el apartado de resultados, pretendían reproducir una escena actual, pasada o reparatoria. En cada ejercicio se conjugaron varias de las siguientes técnicas psicodramáticas (Reyes, 2005), intervenciones verbales como señalamientos, interpretaciones o confrontaciones; no verbales como maximización, focalización, concretización y escultura; y otros recursos como el uso del ego auxiliar mediante el doble, espejo, inversión de roles y soliloquio.

Cada sesión grupal tuvo una duración de 2 horas y se efectuaron una vez por semana, mediante co-terapia (hombre - mujer). El terapeuta asumió la conducción principal y la dirección de los ejercicios psicodramáticos; mientras tanto, la coterapeuta se encargaba de la observación y el registro escrito, así como también de intervenciones terapéuticas. En suma, se realizó una sesión individual con cada uno al inicio (entrevistas preliminares), 8 sesiones de psicoterapia a nivel grupal y una sesión de seguimiento un mes después.

En relación con las "entrevistas preliminares" en psicoanálisis (Miller, 2006; Aulagnier, 2003; Braier, 1999), se realizaron 2 momentos preliminares al dispositivo terapéutico; es decir, primero se efectuaron entrevistas individuales semiestructuradas (Anexo 1) por medio de una guía de preguntas para conocer su demanda inicial, historia de vida y posible estructura clínica como medio de verificación de los criterios de inclusión.

El segundo momento se dio con las personas seleccionadas durante la sesión inicial del grupo al encuadrar el proceso y continuar indagando sobre la demanda, la historia de violencia, la relación con el Otro y, siguiendo a Aulagnier (2003), la potencialidad de análisis en el encuentro con lo grupal. Este segundo momento se acerca más al "tratamiento de ensayo" de Freud (1913/1976), el periodo en el que buscaba definir el diagnóstico diferencial del sujeto para dirigir la cura y si es apto para participar del proceso psicoterapéutico. 
Recolección de la información, sistematización y análisis

Se utilizaron entrevistas semiestructuradas en las sesiones individuales (entrevistas preliminares) y en la post-sesión (evaluación), además de observaciones participantes y grabaciones de audio de las sesiones grupales. La observación pretendía registrar sucesos, eventos e interacciones (Sampieri et al., 2014) y el comportamiento verbal y no verbal (Barker et al., 2002).

Para sistematizar el trabajo de cada sesión, se elaboraron tablas y estructuras esquemáticas. Se registró la descripción de los hechos, intervenciones terapéuticas, reacciones/resultados en los participantes e interpretaciones de los terapeutas, así como hipótesis clínicas, de acuerdo con el registro de observación elaborado (Anexo 2). Adicionalmente, se realizaban preguntas centrales al cierre de cada sesión para evaluar el proceso a nivel de insight.

La transcripción de las sesiones se efectuó en el momento posterior a estas, paralelo a la guía de observación y se procedía a eliminar los audios. Posterior a cada una, el equipo terapéutico compartió un análisis de los elementos transferenciales y contratransferenciales en un estilo de "conversación en caliente" (Anexo 3).

Se contó con un consentimiento informado avalado por el posgrado en psicología que sigue los lineamientos indicados por el Reglamento Ético Científico de la Universidad de Costa Rica para las investigaciones en las que participan seres humanos (Versión Junio 2017). Este contempló la información, voluntariedad y confidencialidad del proceso, la autorización para grabar las sesiones, las cuales serían eliminadas una vez transcritas y para el uso de seudónimos.

Un mes después del proceso se desarrolló una sesión grupal de seguimiento que ofreció a los participantes evaluar y asimilar su trabajo terapéutico, mediante una serie de preguntas abiertas que se discutieron libremente (Anexo 4). Finalmente, se evaluó el proceso grupal, se caracterizó y evaluó cada sesión junto con sus hipótesis clínicas y se realizó una descripción clínica de los participantes y los tipos de violencia experimentados.

El tratamiento de los datos se realizó por medio de análisis por categorías, el cual consistió en identificar núcleos temáticos en el discurso de los participantes durante las entrevistas y el espacio de psicoterapia grupal, así como de las intervenciones realizadas por parte del equipo terapéutico (Arévalo, 2009) y sus efectos. Por tanto, las categorías de análisis fueron:

1. "Historias de violencia en la infancia" corresponde a la descripción de los hechos vividos por cada uno de los hombres.

2. "Influencias de la vivencia infantil en la adultez" guarda relación con el significado de la violencia y el efecto en los comportamientos de estos hombres.

3. "Aportes terapéuticos: la palabra y el cuerpo" consiste en la incidencia de la labor terapéutica como de la transferencia y elaboración grupal.

De las entrevistas individuales y los textos transcritos de las sesiones grupales, se leyó y distribuyó el discurso verbal como no verbal (observaciones) de los participantes y equipo terapéutico en las categorías correspondientes para poder describir el proceso realizado. Por último, se procedió a la unificación, revisión y análisis por cada categoría de toda la información obtenida, por medio de la triangulación de datos entre los distintos medios utilizados, entre el equipo terapéutico-investigador y entre este y el equipo asesor del estudio. 


\section{Resultados}

El material sistematizado se revisó en función de una serie de categorías de análisis que se extrajeron a partir del discurso de los participantes del grupo, como también de las intervenciones efectuadas desde el equipo terapéutico. Por tanto, los resultados abarcan la historia de violencia infantil y la influencia de la misma en la adultez de estos hombres, así como los aportes terapéuticos en el dispositivo grupal.

Historias de violencia en la infancia

El proceso terapéutico permitió identificar elementos característicos del tipo de maltrato infantil que estos hombres tuvieron por sus figuras parentales. Si bien dicho maltrato se manifestaba de forma física en ellos, también solía presentarse de manera psicológica con o sin agresiones corporales (Tabla 2), como se encuentra en distintos estudios (Naciones Unidas, 2019; Ministerio de Salud, INEC., \& UNICEF, 2018; UNICEF, s.f.). De este modo, manifestaron haber recibido actitudes de rechazo y negligencia por parte de aquellas figuras: ausencia física, verbal o bien afectiva, padres presentes pero ausentes emocionalmente o bien totalmente ausentes, a la vez que hacían referencia a un contacto físico más bien agresivo. "Yo también viví eso, agresiones, un papá ausente, una madre controladora" (Javier).

A partir del discurso de estos hombres, la madre se convierte en una figura ambivalente, de modo que resulta difícil de integrar la figura de la que se espera amor y cuidado (en unos se daba y en otros no) con la que se recibe agresividad en la forma que ejerce disciplina. "Es muy cariñosa, pero si se ponía violenta... tiraba algo y si había algo que no le gustaba..." (Antonio).

Es posible que estas actitudes y ambivalencias pudieran afectar su forma de vincularse, la toma de decisiones y la búsqueda de aprobación, como se evidencia más adelante, ya que el deseo de sus padres se mostraba excesivo e insaciable sin poder cumplir sus expectativas: "Me dijeron 'está mal hecho', 'sos un inútil', 'no servís para nada'... me vine a dar cuenta de ese fantasma que no me dejaba avanzar" (Iván).

Ya no solo se ignora o se deja de hablar, sino más bien se registra una palabra, pero violenta; allá donde la falta/ la ausencia era agresión, ahora la expresión también lo es y delimita la acción. Mediante el trabajo terapéutico fue evidente las marcas que este tipo de frases y etiquetas hicieron en torno a la seguridad, la confianza, la toma de decisiones y su autoestima.

Tabla 2

Tipos de violencia experimentada

\begin{tabular}{|c|c|c|}
\hline Manifestaciones & Características & $\begin{array}{c}\text { Cantidad de participantes } \\
\text { afectados }\end{array}$ \\
\hline Psicológica & $\begin{array}{l}\text { Violencia verbal, ofensas, amenazas, abu- } \\
\text { so de autoridad en la toma de decisiones, } \\
\text { disminución del autoestima y valor. }\end{array}$ & 9 casos \\
\hline Física & $\begin{array}{l}\text { Nalgadas, manotazos, chancletazos, } \\
\text { fajazos. }\end{array}$ & 9 casos \\
\hline Brutal/grotesca & $\begin{array}{l}\text { Cachetadas, golpes con objetos como pa- } \\
\text { los, escobas u otros artefactos, lesiones } \\
\text { corporales en ocasiones con sangrados. }\end{array}$ & 5 casos \\
\hline Sexual & Abuso sexual & 2 casos \\
\hline
\end{tabular}

Actualidades en Psicología, 35(130), 2021, 75-95 
Ahora bien, puede decirse que el maltrato psicológico acompañado de agresión corporal es la combinación que asegura el cerrojo, si funciona la analogía en relación con sus dificultades emocionales. Entonces, no solo se da rechazo, sino que allí donde se demanda afecto, se da otra cosa, se inscriben palabras y golpes en el cuerpo como única posibilidad de recibir algo en algunos de estos casos, el único lazo posible con el otro: "Fajazos, chancletazos o con lo que tuviera en la mano" (Mateo); "Patadas, cachetadas, no violencia sexual, en mi caso. Tal vez lo normalicé, pero era bastante real" (Isaac).

Los episodios de agresiones corporales se realizaban ya sea como métodos de castigo o como formas de descarga emocional; referían actos punitivos intensos, impulsivos y, en algunos casos, desproporcionados con respecto a la conducta "sancionada" (Caja Costarricense de Seguro Social, 2012). Ante la voracidad de estos actos, surge un deseo de huida al haber una suerte de ser tomado como objeto por el otro, mientras que paradójicamente era la única forma de ser alguien para estas figuras de crianza. Tres hombres abandonaron la casa a muy temprana edad. "Desde los 11 años que me fui de la casa, no pude enderezar mi vida" (Rafael).

Los que se quedaron reconocen que no había nada que pudieran hacer ante los eventos de un otro tan devorador. Ante el miedo y la impotencia, asumían una posición de silencio y resignación, se colocaban como una tortuga (Mateo), con un caparazón o armadura ( Leonardo), para protegerse de la agresión vivida en la infancia como del dolor y el miedo en la adultez: "Cuando uno es niño, uno no puede correr, uno lo que siente es miedo, uno se resigna" (Antonio).

Esta colocación impuesta los lleva a aceptar ese lugar desde muy pequeños, al punto de justificar la violencia recibida. Se da una suerte de negación: "Es que fue huevonada mía... cómo no le ayudé" (Antonio); "Yo ahora veo chamacos y no se pienso que tal vez les falta faja (...). A ella le pegaron también de niña" (Mateo).

Existe una validación y justificación de lo que es considerado como "castigo", de forma que se normaliza y resta importancia; no se ve como violencia sino disciplina. Se interpreta como algo que se merece. Asimismo, expresaban culpa y merecimiento del castigo recibido, de modo que se les dificultara cuestionar a la figura que ejercía el maltrato (Miller, 2009).

\section{Influencias de la vivencia infantil en la adultez: experiencia subjetiva}

A lo largo del proceso grupal, se fue encontrando también una serie de conflictos en su vida actual con relación a elementos emocionales como comportamentales (Tabla 1). Por ejemplo, en los participantes se presentaba la dificultad de reconocer y expresar sus emociones (Campos \& Salas, 2002). Se les escuchaba decir que no podían derramar una lágrima, no sentían nada o bien lo ocultaban; no lograban conectar con lo que sentían o huían de situaciones que implicaban tener que enfrentar alguna emoción. "En mi caso, en veces he sentido que no siento nada" (Rafael).

La forma violenta en que las figuras de crianza se han vinculado con estos sujetos guarda relación con los mandatos sociales de la masculinidad tradicional; no se les ha validado ni permitido la expresión asertiva de sus emociones. Por tanto, no sorprende que se les dificulte reconocer lo que sienten, pero resulte sencillo aceptar que se siente enojo o ira. "Un sentimiento que me sale es ira, he camuflado mucho, me cuesta identificar sentimientos, porque es lo que sale primero" (Camilo).

El espacio terapéutico permitió identificar otra emoción subyacente: el miedo. Dicho descubrimiento resultó el darse cuenta cómo les había afectado. "Siento miedo, terror, 
pesadillas... en mi cabeza, pasa todo lo que vi, lo que escuchaba, trato muy mal a todos, a los que más amo, se me sale el hijueputa" (Isaac).

El enojo sentido los ha llevado a la repetición de la violencia, mientras que el miedo a la evitación de situaciones que interpretaran como amenazantes. No obstante, esto significaba malestar por no actuar como debían hacerlo según los encargos de la masculinidad, por ejemplo, ser autosuficiente; el conflicto deviene cuando se sienten dependientes del otro: "Mi mamá siempre era la que tomaba las decisiones por mí (...). Me cuesta mucho tomar decisiones, me da miedo, trato de evadir..." (Camilo).

Algunos significantes que los hombres traían para dar cuenta de su actuar y su malestar fueron: procrastinación, zona de confort, autosabotaje. Señalaron, por ejemplo, sentirse cómodos en la incomodidad: "Eso me resuena, lo de moverme. Yo me quedo mucho en la zona de confort, donde no me siento bien. Me siento muy cómodo en ciertas posiciones" (Isaac).

Para ellos, el autosabotaje era una forma de impedirse alcanzar lo que se quiere, reproduciendo la limitación y prohibición que sentían en su infancia, ahora por ellos mismos. "Yo tengo una vara que me jode mucho, es el autosabotaje, me cuesta tomar decisiones, por ende, me quedo atascado" (Isaac).

Además de la dificultad de poder tomar decisiones y acciones, también resaltaron muchas veces la búsqueda de aprobación y aceptación del otro, así como cumplir sus expectativas. "Hace poquito me separé, y estoy aprendiendo a no cumplir las expectativas de otros (...) yo en mi adolescencia hacía todo por mi familia, me casé e hice lo mismo, siempre buscando la aprobación de todo mundo" (Camilo).

Más adelante pudieron identificar que no consideraban merecer nada positivo, autoevaluándose de forma negativa y negándose el disfrute." No merezco nada mejor que esto (...) Ni me responsabilizo, ni me valoro (...) era un sentimiento de aprisionamiento, de reducción como persona" (Francisco).

Todo lo anterior no solo afectó sus proyectos personales, sino también sus vínculos con los demás y consigo mismos. De esta manera, han ejercido comportamientos agresivos hacia otras personas, especialmente, dentro de la familia:"Pero también está la otra parte, me dan celos... Sigo haciendo daño, termino pidiendo disculpas, mucho de ese veneno que pasa por mi boca... y luego pido disculpas... El David Banner de los ochenta, el Hulk. Cuando me cagué en todo, rompo cosas" (Isaac).

Hacia sí mismos, se evidenciaron comportamientos autodestructivos directos e indirectos (Clemente \& González, 1996). En relación con los segundos, se identificaron conductas de transgresión y riesgo, descuido de la salud, negligencia personal y social, falta de planeamiento y pasividad e impotencia; esta última se refiere a evitar o abandonar actividades de involucrarse o tomar acciones, como ya se vio (Tsirigotis et al., 2013). En los actos directos, se encontró en ellos ideaciones e intentos suicidas a lo largo de la vida. "Yo desperdicié mi vida, desde los 15 años en drogas, paré el colegio, y de ahí en adelante nada (...). La herida está sangrante, cuando tenía 20 me intenté suicidar" (Leonardo); "Tengo adicciones, me considero alcohólico, fumo mota... y a veces sí siento que me ha afectado, tener patrones autodestructivos, emborracharme, hacer cosas peligrosas, que no he estado preocupado por mi vida, por mi cuerpo, por mi salud" (Mateo).

En síntesis, en la vida adulta de estos hombres ha existido una serie de formas de violencia que han reproducido en otras personas como en sí mismos, de forma directa e 
indirecta. También, se encontraron manifestaciones de impulsividad y falta de regulación en sus actos y emociones, siendo frecuente los problemas de drogas e ideación suicida en la mayoría de los casos, así como la presencia de violencia física o emocional en las relaciones de pareja.

\section{Aportes terapéuticos: la palabra y el cuerpo}

El rumbo de la intervención acudió a señalamientos que apuntaron a localizar al sujeto en la experiencia grupal y dimensionar el peso de las figuras cuidadoras en la construcción subjetiva; se trajo a colación el conflicto del deseo y la desilusión por la mirada de los padres. Las intervenciones e interpretaciones respecto al discurso de los participantes, permitieron subrayar la posición de cada uno de ellos en cuanto a su historia de maltrato y vida actual.

Las técnicas corporales y psicodramáticas (Reyes, 2007; Rache, 2015) apoyaron la escucha psicoanalítica, permitiendo que la palabra atravesara el cuerpo. Cuando la palabra parecía no moverse de lugar, se realizaba alguna de estas técnicas, utilizando algún significante que se haya señalado. A continuación, se brinda un resumen de cada una de los ejercicios realizados:

El ejercicio 1 fue una actividad entre lo estático y el movimiento, mediante esculturas y maximizaciones; se representó la impotencia vivida en la niñez colocando al protagonista en el centro de la escena y el resto de hombres alrededor de forma rígida como estatuas imposibles de mover. En lo verbal y lo corporal se abordó la violencia física y la negligencia, búsqueda de aprobación, manifestación de la ira y la escasa valoración.

El ejercicio 2 fue una secuencia de dos momentos (infancia y vida adulta) que dan sentido a sus posturas corporales según lo narrado. Se profundizó en la violencia y las emociones sentidas, se maximizó gestos como arquear la espalda y comprimir el cuerpo como protección, y simbolizando la postura y qué emociones encierra. Se pasó de la palabra a lo corporal y viceversa (Arévalo et al., 2016). El terapeuta colocó el brazo sobre el protagonista y, como ego auxiliar, instaló reflexiones que permitieron apalabrar los gestos corporales: "Antes no me podía defender, ¿y ahora? me pongo violento, agresivo, enjachador... chistoso, analítico, ser buena nota (como defensa)" (Terapeuta).

El ejercicio 3 combinó lo corporal (la postura y la fuerza) con lo verbal (repetición de frases en voz alta), de modo que los compañeros presionaban al protagonista hacia abajo, mientras repetían frases recibidas en la infancia. Algunos señalaron que se vieron ahí mismo, identificados con el discurso y la posición tomada. Resultó interesante que Isaac dijo sentirse cómodo sosteniendo la fuerza física de los demás, aduciendo que estaba acostumbrado. "Aguantar, aguantar, reprimir... gritos, ofensas, maltratos, hago de esto mi vida" (Terapeuta).

El ejercicio 4 era realizar un círculo en el cual el centro, adentro, era aquello temido, mientras los bordes la zona de confort, lo conocido. Se dieron cuenta que aún en esa zona siguen recibiendo "golpes", como si estuvieran aún a la espera de recibir un castigo y el miedo no les permitía avanzar al haber asumido esa posición de víctimas y dependientes de los demás. "Yo he resumido que lo que tengo es miedo de avanzar, ser feliz, de tener cosas nuevas... miedo que si nadie me quiere, yo destruyo... pero sin embargo ... una parte de mi dice 'deme', 'golpéeme'" (Iván).

Para el ejercicio 5 se formó un círculo alrededor del protagonista, diciéndoles "esta es la prisión en la que viven"; mientras relataban con soliloquios lo que aquel estaría sintiendo, iban 
cerrando más el círculo. Se preguntó qué están ganando en esa posición y por qué bloquean la salida; se denota la intención de representar el atrapamiento de sí mismos como acción punitiva que contiene. Después se modificó con frases positivas para reparar la escena.

El ejercicio 6 pretendía representar la dualidad de la figura materna, que causa dolor y a la vez mucho respeto. Se pidió tratar de representar esa figura e integrarla en una sola escultura. El abordaje exploró la dificultad de integrar ambivalencias de las figuras cuidadoras. Concluyeron que aún seguían buscando una figura de amor idealizada a la que se aferran, quedando atrapados en relaciones de dependencia y violencia.

El ejercicio 7 fue de esfuerzo físico y visualización. Los participantes hicieron una "fila india" donde cada uno jalaba hacia atrás al de adelante. Se preguntó cómo se sentían, qué o quién representaba el de más atrás (el niño que fueron) y los de adelante (sus etapas de vida). Se interiorizó qué parte de esa historia los sigue deteniendo y a preguntarse (al niño) qué quería o quiere aún (Herrera, 2009). Se analizó cómo el pasado jala hacia atrás y no deja avanzar. Se fue reelaborando un desplazamiento del malestar y la culpa. "Perdonarme por la necesidad de ser perdonado... no ser tan duro conmigo mismo" (Isaac).

El ejercicio 8 implicaba movimiento, concentración y seguir instrucciones entrelazados en círculo. Se dieron cuenta que aún siguen instrucciones confusas que causan "cortocircuito", posicionándose como ese niño recibiendo mensajes contradictorios y violentos.

El ejercicio 9 consistió en representar una escultura del miedo (o herida abierta) mediante el cuerpo, sentir y observar sus posturas. Después debían deconstruir esa imagen y formar una reparadora. Al "dar movimiento" a esa escultura, cambiaron de posturas comprimidas, agachados y con el rostro oculto, a posturas abiertas, miradas tranquilas o abrazándose.

El ejercicio 10 buscaba hacer conexión emocional con ellos mismos, de modo que cada uno pudiera darse un abrazo y permitirse sentir ese contacto consigo mismos. Permitió realizar un cierre emotivo con lo que sintieron a partir de ese encuentro con sus emociones desde una posición distinta integrando todas las imágenes propias que abrazan estos hombres.

El dispositivo facilitó dar cuenta de la relación violenta y negligente con ellos mismos y con los demás y el lazo que guarda con su propia historia. Dicho insight permitió la elaboración y transformación de sus comportamientos en prácticas de autocuidado y de vínculos más sanos con el otro; se dio un desplazamiento de lugar y de movilización del deseo. "Me motiva cada vez más a sacar todo eso que hay ahí, a cambiarlo y a lograr una vida mejor... para mejorar una relación con los otros, primero necesito mejorar conmigo mismo" (Leonardo); "Fui al mall, al cine, me compré ropa, comí, me senté a ver la gente pasar, disfruté mucho, un tiempo para mí" (Antonio).

Fueron elaborando la experiencia subjetiva al apalabrar el dolor vivido y resignificarlo a través del cuerpo. El reconocerse como sujetos con una serie de aspectos positivos y herramientas emocionales les permitió hacerse preguntas y descolocarse del lugar de malestar, a través de la resonancia grupal y la validación de la experiencia vivida.

\section{Discusión}

La intervención clínica efectuada denotó que los participantes experimentaron violencia de distintas formas durante su infancia, lo cual generó un impacto en su vida adulta. Siguiendo a Rosabal (2009), se denotó un estilo autoritario en las figuras de crianza, 
quienes ejercían control y provocaban culpa en ellos. Fernández (2001) señala que el poder de esta época es el poder disciplinario en torno al cuerpo y al control del deseo; por tanto, la agresión y negligencia también podrían ser consideradas como formas de control en estos hombres.

El discurso de ellos evidenció la normalización, aceptación y justificación de esas conductas ejercidas por sus figuras de autoridad mediante el ejercicio del poder y del miedo, además de reproducirlas en sus hijos e hijas, lo cual coincide con Miller (1998; 2002; 2009). También, expresaron el sentimiento de culpabilidad ante el castigo físico (Miller, 2009; Patronato Nacional de la Infancia, 2016), experimentan culpa al hablar sobre el acto recibido, ya que implicaba cuestionar a quien lo ejerció. Habían guardado reclamos para no sentirse culpables, tapando y sosteniendo la falta del Otro (Lema, 2014).

Para estos hombres, la relación con sus figuras parentales, en especial la materna, representa un conflicto al no poder integrar la madre afectiva y cuidadora, y la madre violenta, pero incuestionable; se demandaba amor y se recibía rechazos y golpes, o bien amor y golpes. El maltrato como forma de recibir algo parece el lazo imaginario posible con esa figura para poder sostenerse (Uribe, 2010). No encontrar sentido ante la violencia lleva al sujeto a llenar con un discurso más tolerable ese espacio vacío como interpretación del castigo, acabaron justificando a los padres y culpándose a sí mismos (Miller, 1998), velaron la pregunta sobre el deseo del Otro (Uribe, 2010), pero no el dolor.

El valor que tiene la madre en ellos como figura ambivalente atañe a la construcción histórica del género femenino instalado en la mirada del patriarcado (Butler, 2007). Ante dicha construcción, resulta interesante que, en la mayoría de estos casos, estas madres estaban solas; no había una figura, un tercero que regulara el goce, como diría Lacan (1932, citado en Lema, 2014), mientras que en otros el acto voraz era reflejo de la violencia recibida por la pareja.

En este sentido, el vínculo que estos hombres han tenido con el otro y con su propio cuerpo es de miedo y de protección. Se conjuga la pulsión de vida y de muerte (Freud, 1920/1976), de huida y de ataque como sobrevivencia, sin tener las herramientas psíquicas adecuadas para actuar según la amenaza. Una primera consecuencia de esto es el congelamiento, como el caso de la dificultad en la toma de decisiones, la dependencia, la procrastinación, el autosabotaje, la búsqueda de aprobación y de cumplir las expectativas del otro. No obstante, entran en crisis al no cumplir y dar prueba de virilidad y autosuficiencia (Gilmore, 1994).

Si como indica Cuervo (2010), el apoyo y el afecto permiten un desarrollo cognitivo y psicosocial adecuado en la infancia, el castigo físico y la negligencia pudieron afectar en dicho desarrollo ligado a la aceptación de las figuras significativas. Aquella violencia se instauró consumando una fragilidad yoica (Uribe, 2010), lo cual afectó sus recursos para enfrentar problemas, tomar decisiones, adaptarse a los cambios y valorarse a sí mismos, es decir, las herramientas psicoafectivas que señalaran Rosabal (2009) y Cuervo (2010).

El resultado ha sido la reproducción de medidas defensivas y evitativas que no les ha permitido moverse de lugar a nivel simbólico al no contar con el desarrollo emocional necesario. Hay algo en lo real que irrumpe (García, 2005), que da miedo y detiene, de modo que se protegen de cualquier acto que pudiera ser amenazante para su yo frágil, tal es el caso mediante la procrastinación, como forma de aplazar el deseo. A pesar del precio de displacer, estos sujetos se cuidan de algo que interpretan aún más displacentero (Freud, 1920/1976). 
Una segunda consecuencia ha sido la reproducción de la violencia, de manera que sostienen una relación de maltrato consigo mismos (Hardt et al., 2008; Evren et al., 2011; Carlson et al., 2013). No solo se evidencian estos actos autodestructivos indirectos que se resumen en la pasividad e impotencia que indicaban Tsirigotis et al. (2013), como repetición de lo sentido en la infancia, sino también a conductas de riesgo y la falta de autocuidado de la salud (Badinter, 1993; Salas, 2005; Butler, 2007). Asimismo, el uso de drogas y los intentos de suicidio confirman este vínculo negativo con el propio cuerpo.

Así, se da una compulsión a la repetición (Freud, 1920/1976), donde la pulsión regresa una y otra vez sobre estos sujetos. Se reproduce nuevamente el trauma, como algo excesivo que reaparece sin motivación (Lacan, citado en García, 2005): el castigo por parte de las personas cuidadoras, pero ahora por su cuenta, de forma que el objeto pasa a ser el propio yo. El deseo de destrucción se redirige hacia ellos mismos en lugar de un objeto externo que no puede ser atacado (Freud, 1915-1917/1976), las figuras parentales causantes de dolor (Miller, 1998; Miller, 2009). Miller (2009) señala, precisamente, que dicha experiencia permanece guardada en la memoria corporal y emocional que le llevará a repetir el acto contra sí, lo cual refuerza la necesidad de un abordaje en torno al cuerpo.

Asimismo, en el discurso de estos hombres se refleja el aprendizaje social por medio de los mandatos de la masculinidad hegemónica que dictan ser fuertes, ser valientes, defenderse, no mostrar las emociones (Salas, 2005; Campos \& Salas, 2002). Esto parece haber significado una herramienta o una vía para tramitar en el cuerpo el trauma vivido, aun cuando se transforme en daños hacia ellos y otras personas. En efecto, desde la masculinidad tradicional resulta censurable expresar las emociones, salvo el enojo, mientras que se censuran las demás como el miedo o la tristeza, a la vez que socialmente se validan las conductas agresivas. Frente a esto, brindar un espacio para la palabra y para la escucha pudo permitir la queja y denuncia que había sido censurada desde muchos frentes como la normalización de la violencia disfrazada como disciplina, justificación de los padres y una masculinidad hegemónica.

Contar con una audiencia que escucha, no censura y valida el dolor, sirve de eco y espejo. Freud (1921/1976) señaló que el fin de la identificación es configurar el yo frente a otro como modelo, por cuanto el emergente dentro del grupo permitió un encuentro con otro especular, lo imaginario (Herrera, 2009; Nominé, 2004), donde pudieron escucharse a sí mismos a través de la identificación y el vínculo con el otro y su relato o la escena que protagonizaba. Siguiendo a Kesselman, la escena individual conlleva la identificación grupal (1996, citado en Reyes, 2007).

Lo anterior se promovió el verse y escucharse desde otro lugar distinto al que habían sido colocados desde su infancia y, por tanto, se resignifica la experiencia subjetiva como también la palabra que se va diciendo conforme es escuchada. Asimismo, posibilitó que estos hombres pudieran movilizarse subjetivamente, en términos de Romero y Sauane (1999), y apostarse por el deseo; esto por cuanto, aun desde el silencio, escuchar y ser espectador puede tener efectos significativos en el sujeto que se mira en el espejo.

El síntoma fue dicho por medio del lenguaje y tuvo la posibilidad de ser escuchado por él mismo, el grupo y el equipo terapéutico. Por tanto, no solo este último fungió de testigo sino el grupo mismo, el cual facilitó y escuchó la denuncia y la queja de cada uno. En este encuentro de subjetividades donde aparece la transferencia (Freud, 1913/1976; Reyes, 2005), se produjo el espacio para una nueva escucha, por parte de los terapeutas 
(hombre y mujer). Así, teniendo cuidado de los lugares transferenciales de las figuras de infancia del sujeto, como lo plantea Aulagnier (2003), se intentó plantearles cómo es posible estar en otro lugar.

Se posibilitó una libre circulación de la palabra del sujeto y una lectura más allá de la superficie del síntoma expresado y de la demanda; se trató no solo de no taparlos sino de destapar algo más que subyace permitiéndoles hablar (Freud, 1913/1976). Aunque la demanda del grupo era hablar de lo actual, era preciso hacer la asociación con lo vivido, con lo traumático (García, 2005); había resistencia de revisar la niñez, como si se quisiera trabajar su infancia sin tocarla, debido al dolor. Haciendo eco de Aulagnier (2003), se debía analizar la relación del yo con el ello, el yo con el "antes" y los objetos de la demanda actual; así, siguiendo dicha autora, se logró desmembrar las voces de figuras fantasmáticas de la infancia y reconocer la voz de sí mismos, para deshabitar discursos avasalladores.

Más aún, se apuntó a encontrar el vínculo con la memoria no solo desde el lenguaje verbal sino también desde la dimensión de lo corporal (Miller, 2009; Reyes, 2005); se dio espacio para la lectura de dos tipos de textos, el discurso y el cuerpo. El primero se refiere a lo verbal, mientras que el segundo implica que si se le permite al cuerpo expresarse se puede encontrar con un discurso y una historia, no verbal, en función de un síntoma (Fernández, 2001; Lema, 2014; Soler, s.f.). Se coincide con Lacan (Nominé, 2004; Soler, s.f.) sobre la construcción del cuerpo desde lo simbólico y lo imaginario, por lo que resultan clave en la práctica psicodramática psicoanalítica (Herrera, 2009) y la relación entre lenguaje y cuerpo al abordar experiencias traumáticas (Lacan, 2012), donde está el agujero, donde no hay palabras.

Arévalo et al. indican que "con el relato, la reflexión surge de un proceso cognitivo; con la corporalidad, la reflexión surge del movimiento y la escenificación en un juego de roles" (2016, p. 233). Surgieron así las voces del pasado y los recuerdos, así como la disposición de dejarse observar y escuchar por el otro, de movimiento y complicidad, gracias a la transferencia, en tanto repetición (Freud, 1914/1976) y depositación en el otro; afloró la memoria corporal y emocional de lo que habría estado reprimido (Reyes, 2005), o bien había estado consciente, pero sin poder apalabrarlo.

Se dio un juego que pasa de la palabra al cuerpo y del cuerpo de nuevo a la palabra, mediante los recursos psicodramáticos que permiten las expresiones corporales y verbales (Reyes, 2007; Rache, 2015). Estos surgieron a partir del relato de los hombres y de la lectura e interpretación del terapeuta (Freud, 1913/1976), como forma de evocar la vivencia y visualizar lo que no era consciente; podían mirarse desde otra posición por medio del movimiento y al volver al discurso moverse nuevamente, con otra escucha y con otra posibilidad de resignificar la experiencia vivida (Herrera, 2009). Se coincide con Rache (2015) en poder simbolizar la apropiación del sujeto en acto, sin quedar solo en la palabra o en el cuerpo, sino en acto.

Si bien las expresiones corporales solían ser de tipo esculturas (sin movimiento) (Reyes, 2007), entre otros recursos psicodramáticos, al plantear una palabra o una pregunta, un cambio de escena o de protagonista, se generaba otro sentido de la escena traumática y, por tanto, otra forma de mirarla, apalabrarla y escucharla; entonces se mueven. Se convirtió en una especie de Ford-da (Freud, 1920/1976) en el que no solo hay un ir y venir de la palabra al cuerpo y viceversa, sino que ahora hay un rol activo del sujeto en el "juego"; se repite una acción que fue dolorosa y quedó atrapada en el cuerpo, permitiendo ahora poder tramitarla para dejarle transitar libre y activamente.

Actualidades en Psicología, 35(130), 2021, 75-95 
Asimismo, se abrió la posibilidad de conectarse con el cuerpo y con las emociones que no habían podido explorar, debido a la dificultad de reconocer, identificar y verbalizar (Salas, 2005; Campos \& Salas, 2002). Cabe resaltar cómo los participantes recordaban con base en los ejercicios realizados para hacer referencia a la sesión anterior; por cuanto, el movimiento, lo corporal, permiten una memoria y una mirada distinta para resignificar la experiencia y traerla a la conciencia. La forma de memoria que resume una experiencia nueva y moviliza el deseo no es más que este juego donde la palabra encuentra al cuerpo y viceversa.

De esta forma, este estudio logró tomar herramientas de la psicoterapia grupal, como el compartir la experiencia y la identificación con el otro, además de elementos del psicodrama como el uso del cuerpo, y del psicoanálisis, la escucha activa y la asociación de ideas, con el fin de trabajar una temática muy excluida en hombres. Se difiere, en este sentido, de otras prácticas que dictan un abordaje exclusivo del discurso verbal, solo individual o que buscan anestesiar o eliminar en el cuerpo las sensaciones y emociones sin dar voz a lo vivido. Resulta importante un método que dé cuenta del sujeto, del valor de la palabra y de las identificaciones transferenciales, pero también de ese cuerpo hablado, que permita la resignificación y un cambio de posición.

Este trabajo constituyó una sistematización de un proceso terapéutico grupal, la cual cobra valor al no haber investigaciones y sistematizaciones en grupos de hombres en Costa Rica, en especial abordando la violencia vivida en la infancia, a pesar de realizarse muchas intervenciones en población masculina. La importancia de dicho estudio yace en visibilizar la necesidad del trabajo en hombres que han vivido violencia infantil y que a su vez pueden ser generadores de violencia; asimismo, aporta elementos básicos para su posible abordaje mediante técnicas psicodramáticas y psicoanalíticas como una apuesta de intervención, junto con un enfoque de género y de masculinidades positivas.

Resulta relevante poder profundizar en propuestas como estas que puedan brindar mayores insumos e indicadores en procesos grupales con hombres. No obstante, se reconocen las dificultades prácticas que implica realizar trabajos amplios en temáticas específicas, en especial cuando hay una demanda actual que no pasa por la experiencia traumática necesariamente. Cuando es posible abordarse, es limitado el seguimiento que se le da por la fluctuación que suele haber en los grupos terapéuticos o de apoyo abiertos; sin embargo, podría ser útil en terapias breves de grupos cerrados como lo es a nivel institucional. Se insta a realizar propuestas que den cuenta del sujeto y del trauma vivido, sin dejar de lado el aprendizaje social propia de una masculinidad tradicional que acompaña a estos hombres.

\section{Referencias}

Arévalo, A., Fernández, B., Hidalgo, F., Lepe, Y., Miranda, C., Nuñez, M., \& Reyes, L. (2016). Corporalidades y narrativas docentes: un dispositivo metodológico para la investigación y formación de profesores. Estudios Pedagógicos, 42(4), 223-242. http://dx.doi.org/10.4067/S0718-07052016000500013

Arévalo, J. J. (2009). Metodología Cualitativa. Cuadernos Educativos. Universidad de San Carlos de Guatemala.

Aulagnier, P. (2003). El aprendiz de historiador y el maestro-brujo: Del discurso identificante al discurso delirante. Amorrortu Editores.

Badinter, E. (1993). XY. La Identidad Masculina. Alianza Editorial.

Actualidades en Psicología, 35(130), 2021, 75-95 
Barker, C., Pistrang, N., \& Elliot, R. (2002). Research Methods in Clinical Psychology: An Introduction for Students and Practitioners. John Wiley y Sons, Ltd.

Beutel, M., Michal, M., \& Subic-Wrana, C. (2008). Psychoanalytically-Oriented Inpatient Psychotherapy of Somatoform Disorders. Journal of The American Academy of Psychoanalysis and Dynamic Psychiatry, 36(1), 125-142. https://doi.org/10.1521/ jaap.2008.36.1.125

Braier, E. A. (1999). Psicoterapia breve de orientación psicoanalítica. Nueva visión.

Burin, M., \& Meler, I. (2004). Varones. Género y objetividad masculina. Paidós.

Butler, J. (2007). El género en disputa. El feminismo y la subversión de la identidad. Paidós.

Caja Costarricense de Seguro Social. (2012). Guía de atención a niños, niñas y adolescentes víctimas de abuso. http://www.binasss.sa.cr/guiaabuso.pdf

Campos, Á., \& Salas, J. M. (2002). Aspectos teóricos generales de la masculinidad. Masculinidades en Centro América. Lara Segura Editores.

Carlson, E., McDade-Montez, E., Armstrong, J., Dalenberg, C., \& Loewenstein, R. (2013). Development and Initial Validation of the Structured Interview for Self-Destructive Behaviors. Journal of Trauma and Dissociation, 14(3), 312-327. https://doi.org/10. 1080/15299732.2012.762822

Clemente, M., \& González, A. (1996). Suicidio. Una alternativa social. Biblioteca Nueva.

Comisión Técnica Interinstitucional sobre Estadísticas de Convivencia y Seguridad Ciudadana. (2019). Masculinidades y tendencias delictivas en Costa Rica. Periodo 2015-2018. Observatorio de la violencia del Ministerio de Justicia y Paz.

Cuervo, A. (2010). Pautas de crianza y desarrollo socioafectivo en la infancia. Diversitas: Perspectivas en Psicología, 6(1), 111-121. https://doi.org/10.15332/s17949998.2010.0001.08

Elliott, R. (2011). Qualitative methods for studying psychotherapy change processes. In D. Harper., \& A. Thompson (Eds.), Qualitative Research Methods in Mental Health and Psychotherapy. A guide for students (pp. 69-81). John Wiley \& Sons, Ltd.

Evren, C., Dalbudak, E., \& Evren, B. (2011). Childhood trauma and quality of life among alcohol dependent men. Anatolian Journal of Psychiatry/ Anadolu Psikiyatri Dergisi, 12(4), 245-252. https://www.researchgate.net/publication/287759080_ Childhood_trauma_and_quality_of_life_among_alcohol_dependent_men

Fernández, A. M. (2001). Instituciones Estalladas. Editorial Universitaria de Buenos Aires.

Fondo de las Naciones Unidas para la Infancia. (s.f.). Violencia emocional. http://www. aeped.es/sites/default/files/documentos/entrega_7-violencia_emocional.pdf

Freud, S. (1913/1976). Sobre la iniciación del tratamiento (Nuevos consejos sobre la técnica del psicoanálisis, I). En S. Freud, Obras Completas, Tomo XII. Amorrortu Editores.

Freud, S. (1914/1976). Recordar, repetir y reelaborar (Nuevos consejos sobre la técnica del psicoanálisis, II). En S. Freud, Obras Completas, Tomo XII. Amorrortu Editores.

Freud, S. (1915-1917/1976). Duelo y melancolía. En S. Freud, Obras Completas, Tomo XIV. Amorrortu Editores.

Actualidades en Psicología, 35(130), 2021, 75-95 
Freud, S. (1920/1976). Más allá del principio del placer. En S. Freud, Obras Completas, Tomo XVIII. Amorrortu Editores.

Freud, S. (1921/1976). Psicología de las masas y análisis del yo. En S. Freud, Obras Completas, Tomo XVIII. Amorrortu Editores.

García, G. (2005). Actualidad del trauma. Grama Ediciones.

Gilmore, D. (1994). Hacerse hombre. Concepto cultural de la masculinidad. Editorial Paidós.

Hardt, J., Sidor, A., Nickel, R., Kappis, B., Petrak, P., \& Egle, U. T. (2008). Childhood Adversities and Suicide Attempts: A Retrospective Study. Journal of Family Violence, 23, 713718. https://doi.org/10.1007/s10896-008-9196-1

Herrera, A. (2009). El psicodrama psicoanalítico: una teoría, una práctica, una experiencia. Poiésis, Revista Electrónica de Psicología Social, (18), 1-8. https://core.ac.uk/ reader/268189200

Lacan, J. (2012). Radiofonía en Otros Escritos. Paidós.

Lema, S. (2014). La maternidad como exceso: clínica contemporánea del estrago materno. Un estudio psicoanalítico [Tesis de Maestría, Universidad de la República]. Conocimiento libre Repositorio Institucional. https://www.colibri.udelar.edu.uy/ jspui/bitstream/20.500.12008/4379/1/Lema\%2C\%20Sebastian.pdf

Miller, A. (1998). Por tu propio bien. Raíces de la violencia en la educación del niño. Tusquets Editores.

Miller, A. (2002). La madurez de Eva. Una interpretación de la ceguera emocional. Paidós Contextos.

Miller, A. (2009). Salvar tu vida. La superación del maltrato en la infancia. Tusquets Editores.

Miller, J. A. (2006). Introducción al método psicoanalítico. Paidós.

Ministerio de Salud, Instituto Nacional de Estadística y Censos, \& Fondo de las Naciones Unidas para la Infancia. (2018). Informe de resultados de la encuesta: Encuesta de Mujeres, Niñez y Adolescencia 2018 (EMNA). UNICEF. https:// www.unicef.org/costarica/media/436/file/Encuesta-de-mujeres-ninez-yadolescencia-2018.pdf

Molina, L. (6 de junio de 2019). Los suicidios aumentan en Costa Rica ocultos entre las comunidades. Semanario Universidad. https://semanariouniversidad.com/ especiales/suicidios-aumentan-en-costa-rica/

Naciones Unidas. (30 de julio de 2019). Violencia contra los niños. Informe de la Representante Especial del Secretario General sobre la Violencia contra los Niños. Asamblea General. https://undocs.org/es/A/74/259

Nominé, B. (2004). El cuerpo y la psicosis. Asociación Costarricense para la Investigación y Estudio del Psicoanálisis.

Organización Mundial de la Salud. (8 de junio de 2020). Maltrato Infantil. http://www.who. int/mediacentre/factsheets/fs150/es/

Patronato Nacional de la Infancia. (5 de agosto de 2016). Protocolo para la atención de abuso emocional. http://www.pani.go.cr/publicaciones/documentos/bibliografiarecursos-humanos/582-protocolo-abuso-emocioanl-arreglado-1 
Pavlovsky, C. (1991). La letra de Moreno. En E. Pavlovsky., H. Kesselman., \& C. de Brasi. Colección "Propuestas" (pp. 147-161). Ayllu. https://www.bibliopsi.org/docs/ carreras/obligatorias/CFG/10grupos/percia/primer\%20cuatrimestre\%202020/ Grupos\%20Percia\%202020/GRUPAL\%209.pdf

Poder Judicial. (2019). Suicidios 2018: análisis. https://www.poder-judicial.go.cr/ planificacion/index.php/anuario-policial-2018/file/5112-suicidios-2018-analisis

Rache, E. (2015). Travesía de lo corporal a lo simbólico corporal. Lumen.

Reyes, G. (2005). Psicodrama: Paradigma, teoría y método. Editorial Cuatro Vientos.

Reyes, G. (2007). La práctica del psicodrama. RIL editores.

Romero, R., \& Sauane, S. (1999). Identificación y grupo, perspectiva freudiana. En Grupo, objeto y teoría. (Vol. III). Lugar Editorial.

Rosabal, M. (2009). Violencia en creencias y prácticas de parentaje en el disciplinar: Aproximación desde la psicología del desarrollo. Revista Digital de la Maestría en Ciencias Penales, 1, 389-404. https://revistas.ucr.ac.cr/index.php/RDMCP/article/ view/12638

Salas, J. M. (2005). Hombres que rompen mandatos. La prevención de la violencia. Lara Segura y Asociados.

Soler, C. (s.f.). El cuerpo en la enseñanza de Jacques Lacan. https://agapepsicoanalitico.files. wordpress.com/2013/07/colettesoler-elcuerpoenlaensenanzadejacqueslacan.pdf

Sampieri, R., Collado, C., \& Baptista, M. (2014). Metodología de la Investigación. McGraw Hill.

Tsirigotis, K., Gruszczyński, W., \& Tsirigotis-Maniecka, M. (2013). Gender differentiation of indirect self-destructiveness. International Journal of Occupational Medicine y Environmental Health, 26(1), 39-48. https://doi.org/10.2478/s13382-013-0085-3

UNICEF (s.f). Violencia emocional. http://www.aeped.es/sites/default/files/documentos/ entrega_7-violencia_emocional.pdf.

Uribe, N. (2010). Nuevas perspectivas sobre violencia intrafamiliar. Un enfoque psicoanalítico. Revista Affectio Societatis, 7(13), 1-18. https://revistas.udea.edu.co/ index.php/affectiosocietatis/article/view/7640 


\section{Anexos}

Anexo 1

Registro de entrevista preliminar

\begin{tabular}{|c|c|c|}
\hline Datos personales & Preguntas de la persona entrevistadora & $\begin{array}{l}\text { Criterio de la persona } \\
\text { entrevistadora }\end{array}$ \\
\hline Nombre: & $\begin{array}{l}\text { 1. Aspectos significativos de historia de } \\
\text { vida: }\end{array}$ & $\begin{array}{l}\text { 4. Información adicional relevante } \\
\text { (actitud y comportamiento del } \\
\text { participante, facilidad/dificultad }\end{array}$ \\
\hline Edad: & $\begin{array}{l}\text { 2. Violencia en la infancia: } \\
\text { ¿Quién? ¿Cuándo? ¿Cómo? } \\
\text { ¿Qué piensa sobre lo que pasó y/o qué }\end{array}$ & $\begin{array}{l}\text { para el manejo del grupo, ¿mues- } \\
\text { tra escenario de crisis?) }\end{array}$ \\
\hline Ocupación: & consecuencias tuvo (cómo le afectó)? & \\
\hline Domicilio: & $\begin{array}{l}\text { 3. Expectativas sobre el proceso } \\
\text { terapéutico: }\end{array}$ & \\
\hline
\end{tabular}

Anexo 2

Guía de observación

Descripción de hechos Intervenciones tera- Reacciones/resultados Interpretaciones de principales péuticas

en los participantes los terapeutas

Anexo 3

Aspectos transferenciales y contratransferenciales (conversación en caliente)

\begin{tabular}{ll}
\multicolumn{1}{c}{ Terapeuta } & \multicolumn{1}{c}{ Coterapeuta } \\
\hline Inicio de la sesión: & Inicio de la sesión: \\
Durante la sesión: & Durante la sesión: \\
Después de la sesión: & Después de la sesión: \\
\hline
\end{tabular}

Actualidades en Psicología, 35(130), 2021, 75-95 
Anexo 4

Post-evaluación

Preguntas

1. Experiencia general: ¿Cómo ha sido este proceso psicoterapéutico para usted?

2. Cambios: ¿Cuáles cambios ha notado en usted mismo desde que inició la terapia?

3. Atribución: ¿A qué atribuye estos cambios?

4. Recursos: ¿Cuáles fortalezas o aspectos de su vida le ayudaron a afrontar este proceso?

5. Limitaciones: ¿Qué cosas sobre usted o de su situación fueron limitantes u obstáculos en este proceso?

6. Aportes: ¿Cuáles han sido los aportes de este proceso para usted?

7. Aspectos difíciles:

- ¿Qué tipo de elementos sobre la terapia han sido difíciles, negativos o decepcionantes para usted?

- ¿Hubo algo que le fuera difícil y no pudo trabajar en el proceso?

Nota. Adaptado de Elliott (2011). 